\section{Check for updates}

Cite this: Analyst, 2021, 146, 2152

\title{
Fluorescence sensing of cyanide anions based on Au-modified upconversion nanoassemblies $\uparrow$
}

\begin{abstract}
Chunning Sun (D) * and Michael Gradzielski (DD *
Cyanides have been recognized as one of the most toxic chemicals and are harmful to the environment and human beings. Herein, fluorescence resonance energy transfer (FRET)-based upconversion nanoprobes for cyanide anions have been designed and prepared by assembling Au nanoparticles (NPs) on core-shell-structured $\mathrm{NaYF}_{4}: \mathrm{Yb}, \mathrm{EraNaYF}_{4}$ :Yb upconversion NPs (csUCNPs), where csUCNPs act as the energy donor and Au NPs act as the energy acceptor. The Au content was optimized in order to have a large quenching efficiency in upconversion luminescence (UCL). The cyanide-mediated redox reaction leads to the consumption of Au NPs, resulting in UCL recovery by the inhibition of the FRET process. On the basis of these features, csUCNP/Au nanoassemblies can serve as sensitive nanoprobes for cyanide ions with a detection limit of $1.53 \mu \mathrm{M}$. Moreover, no significant UCL variation was observed upon the addition of other interfering ions, showing the excellent selectivity of nanoprobes toward cyanide ion sensing. The easy preparation of such upconversion-based nanoprobes provides a promising platform for sensitive and selective sensing of other hazardous species.
\end{abstract}

Received 30th September 2020, Accepted 26th January 2021

DOI: 10.1039/d0an01954b

rsc.li/analyst surface-enhanced Raman spectroscopy (SERS) strategies ${ }^{9,10}$ have been developed for the sensing of cyanide ions. Although high accuracy can be achieved by these approaches in quantitative analyses, complicated instruments and time-consuming procedures are usually required. Owing to the high sensitivity, easy operation, and applicability in biosensing, fluorescent chemodosimeters have been widely investigated. ${ }^{11,12}$ Unlike traditional fluorescent materials excited by ultraviolet (UV) or visible light, upconversion chromophores possess different luminescence mechanisms, which are capable of converting near-infrared (NIR) excitation to visible or UV light when codoped with appropriate activator ions. ${ }^{13}$ They feature large anti-Stokes shifts, sharp and narrow multicolor emissions, high chemical stability, and relatively low toxicity. Moreover, the use of NIR light as the excitation wavelength not only enables higher penetration depth but also avoids the biomolecular autofluorescence and background interference. ${ }^{14}$ Hence, upconversion-based nanophosphors have attracted increasing interest in the sensing field. ${ }^{15}$ Generally, the Förster resonance energy transfer (FRET) process is employed for the upconversion-based fluorescence sensing of analytes. Up to now, a number of functional materials have been coupled to upconversion NPs to achieve sensing properties, such as organic dyes, ${ }^{16-23}$ metal-ligand complexes, ${ }^{24-26}$ carbon nanomaterials, ${ }^{27-30}$ noble metals, ${ }^{31-33}$ quantum dots, ${ }^{34-36}$ and two-dimensional materials. ${ }^{37-40}$ Fluorescent chemosensors based on upconversion NPs have been designed for the detection of cyanide ions as well. However, the commonly used organic dyes and metal-ligand complexes, acting as energy 
acceptors, suffer from either photobleaching or complex synthetic processes. ${ }^{41-43}$

In this contribution, we report the facile design and synthesis of nanocomposites through assembling $\mathrm{Au}$ nanoparticles (NPs) on the surface of core-shell-structured $\mathrm{NaYF}_{4}$ : $\mathrm{Yb}$,Er@NaYF 4 :Yb upconversion NPs (abbreviated as csUCNPs). Au-modified csUCNP nanoassemblies (abbreviated as csUCNP/ $\mathrm{Au}$ ) can be applied as fluorescent nanoprobes for cyanide anions based on the FRET mechanism, where csUCNPs act as the energy donor and Au NPs act as the energy acceptor. Moreover, the content of the Au NPs was optimized to achieve maximum quenching efficiency in upconversion luminescence (UCL). The cyanide-mediated redox reaction oxidizes $\mathrm{Au}$ into $\left[\mathrm{Au}(\mathrm{CN})_{2}\right]^{-}$in the absence and presence of other interfering ions, leading to the consumption of Au NPs, inhibiting the FRET process, and thus resulting in the recovery of UCL emissions. Accordingly, these features enable the sensitive and selective sensing of cyanide ions.

\section{Experimental}

\section{Materials}

Yttrium(III) acetate tetrahydrate (99.9\%), ytterbium(III) acetate hydrate (99.9\%), and erbium(III) acetate hydrate (99.9\%) were purchased from Alfa Aesar. Oleic acid (OA, 90\%), 1-octadecene (ODE, 90\%), ammonium fluoride $\left(\mathrm{NH}_{4} \mathrm{~F}, \geq 98 \%\right)$, sodium hydroxide $(\mathrm{NaOH}, \geq 98 \%)$, methanol (99.8\%), ethanol ( $\geq 99.8 \%$ ), $N, N$-dimethylformamide (DMF, 99.8\%), cyclohexane (99.5\%), formic acid ( $\geq 98 \%)$, tetrachloroauric(III) acid trihydrate $\left(\mathrm{HAuCl}_{4} \cdot 3 \mathrm{H}_{2} \mathrm{O}, \geq 99.9 \%\right)$, tetrakis(hydroxymethyl)phosphonium chloride (THPC, $80 \%$ in $\mathrm{H}_{2} \mathrm{O}$ ), $\mathrm{NaCN}(\geq 95 \%)$, and polyethylenimine (PEI, branched, $M_{\mathrm{w}} \sim 25000$ ) were obtained from Sigma-Aldrich. Milli-Q water $\left(18.2 \mathrm{M} \Omega \mathrm{cm}\right.$ at $\left.25{ }^{\circ} \mathrm{C}\right)$ was used in all experiments.

\section{Characterization}

Fourier transform infrared (FT-IR) spectra were acquired with a Thermo Scientific Nicolet iS5 FT-IR spectrometer using the $\mathrm{KBr}$ method. The spectra were recorded in transmission mode in a wavenumber range from 4000 to $500 \mathrm{~cm}^{-1}$. UV-Vis absorption spectra were obtained by using a CARY 50 spectrophotometer. Transmission electron microscopy (TEM) and energydispersive X-ray spectroscopy (EDS) were performed with a FEI Tecnai $\mathrm{G}^{2} 20$ S-TWIN electron microscope with a $\mathrm{LaB}_{6}$ cathode operating at $200 \mathrm{kV}$. Powder X-ray diffraction (XRD) spectra were obtained by using a Philips X'Pert MPD Pro X-ray diffractometer at a scanning rate of $4^{\circ} \mathrm{min}^{-1}$ in a $2 \theta$ range from $10^{\circ}$ to $90^{\circ}$ with $\mathrm{Cu} \mathrm{K} \alpha$ radiation $(\lambda=0.15406 \mathrm{~nm})$. $\zeta$-Potential and dynamic light scattering (DLS) measurements were carried out with an Anton Paar Litesizer ${ }^{\mathrm{TM}}$ 500. UCL emission spectra were collected at room temperature with a fiber-coupled spectrometer (Ocean HDX, Ocean Optics) equipped with an external $980 \mathrm{~nm}$ laser (Roithner Lasertechnik $\mathrm{GmbH}$ ) with a power of 3 W.

\section{Synthesis of Au NPs}

Au NPs were synthesized as described previously. ${ }^{44}$ Typically, $12 \mu \mathrm{L}$ of THPC and $0.25 \mathrm{~mL}$ of $\mathrm{NaOH}(2.0 \mathrm{M})$ were added to $45 \mathrm{~mL}$ of water, and then the mixture was stirred vigorously for 5 min followed by a quick injection of $2 \mathrm{~mL}$ of $\mathrm{HAuCl}_{4}$ solution (1 wt\%). The color changed to dark brown immediately. This solution was stored in a dark container and stirred overnight.

\section{Synthesis of OA-UCNPs}

The synthesis of oleate-capped $\mathrm{NaYF}_{4}: \mathrm{Yb}, \mathrm{Er}$ NPs (abbreviated as OA-UCNPs) was carried out by a high-temperature coprecipitation method according to a previous publication with minor modifications. ${ }^{45}$ Typically, $3.12 \mathrm{~mL}$ of $\mathrm{Y}\left(\mathrm{CH}_{3} \mathrm{COO}\right)_{3}(0.2 \mathrm{M})$, $0.8 \mathrm{~mL}$ of $\mathrm{Yb}\left(\mathrm{CH}_{3} \mathrm{COO}\right)_{3}(0.2 \mathrm{M})$ and $0.8 \mathrm{~mL}$ of $\mathrm{Er}\left(\mathrm{CH}_{3} \mathrm{COO}\right)_{3}$ $(0.02 \mathrm{M})$ aqueous solutions were added to a three-neck flask containing $6 \mathrm{~mL}$ of $\mathrm{OA}$ and $14 \mathrm{~mL}$ of $\mathrm{ODE}$ at room temperature. The mixed solution was heated to $110{ }^{\circ} \mathrm{C}$ for $30 \mathrm{~min}$ to remove the water, followed by heating to $160{ }^{\circ} \mathrm{C}$ for $40 \mathrm{~min}$ to form lanthanide-oleate complexes and then cooled down to $50{ }^{\circ} \mathrm{C}$. A methanol solution $(10 \mathrm{~mL})$ containing $\mathrm{NH}_{4} \mathrm{~F}$ (3.2 mmol) and $\mathrm{NaOH}(2.0 \mathrm{mmol})$ was added afterward and stirred at $50{ }^{\circ} \mathrm{C}$ for $30 \mathrm{~min}$. After evaporating the methanol, the solution was heated to $305{ }^{\circ} \mathrm{C}$ at a ramp rate of $10{ }^{\circ} \mathrm{C}$ $\min ^{-1}$ and maintained at this temperature for 45 min under a $\mathrm{N}_{2}$ atmosphere. After cooling down to room temperature, OA-UCNPs were precipitated out by adding excess ethanol, collected by centrifugation at $6000 \mathrm{rpm}$ for $5 \mathrm{~min}$, repeatedly washed with ethanol, and finally redispersed in cyclohexane.

\section{Synthesis of core-shell-structured OA-csUCNPs}

Oleate-capped $\mathrm{NaYF}_{4}: \mathrm{Yb}, \mathrm{Er} @ \mathrm{NaYF}_{4}: \mathrm{Yb}$ (abbreviated as OAcsUCNPs) core-shell-structured upconversion NPs were prepared by coating a similar material on the core OA-UCNPs. ${ }^{46}$ Typically, $\mathrm{Y}\left(\mathrm{CH}_{3} \mathrm{COO}\right)_{3}(3.2 \mathrm{~mL}, 0.2 \mathrm{M})$ and $\mathrm{Yb}\left(\mathrm{CH}_{3} \mathrm{COO}\right)_{3}$ $(0.8 \mathrm{~mL}, 0.2 \mathrm{M})$ were added into a three-neck flask containing $6 \mathrm{~mL}$ of $\mathrm{OA}$ and $14 \mathrm{~mL}$ of $\mathrm{ODE}$ at room temperature. The mixed solution was heated to $110^{\circ} \mathrm{C}$ for $30 \mathrm{~min}$ to remove the water, followed by heating to $160{ }^{\circ} \mathrm{C}$ for $40 \mathrm{~min}$ to form lanthanide-oleate complexes and then cooled down to room temperature. The above-synthesized OA-UCNPs dispersed in cyclohexane were added to the flask. The solution was heated to $110{ }^{\circ} \mathrm{C}$ to remove the cyclohexane and then subsequently cooled down to room temperature. A methanol solution $(10 \mathrm{~mL})$ containing $\mathrm{NH}_{4} \mathrm{~F}(3.2 \mathrm{mmol})$ and $\mathrm{NaOH}(2.0 \mathrm{mmol})$ was added afterward and stirred at $50{ }^{\circ} \mathrm{C}$ for $30 \mathrm{~min}$. After heating to $110{ }^{\circ} \mathrm{C}$ to evaporate the methanol, the solution was heated to $305{ }^{\circ} \mathrm{C}$ at a ramp rate of $10{ }^{\circ} \mathrm{C} \mathrm{min}^{-1}$ and maintained at that temperature for 45 min under a $\mathrm{N}_{2}$ atmosphere. After letting the solution to cool down to room temperature, OAcsUCNPs were precipitated out by the addition of excess ethanol, collected by centrifugation at $6000 \mathrm{rpm}$ for $5 \mathrm{~min}$, washed with ethanol several times, and finally redispersed in cyclohexane. 


\section{Preparation of ligand-free UCNPs and csUCNPs}

Ligand-free upconversion NPs were prepared via a vortexing method according to our previous publication. ${ }^{47}$ Formic acid ( $5 \mathrm{mmol}$ ) was added to $2 \mathrm{~mL}$ of cyclohexane solution containing OA-UCNPs (10 $\left.\mathrm{mg} \mathrm{mL}^{-1}\right)$ directly; the mixture was then shaken for $10 \mathrm{~s}$ (3000 rpm) on a vortex mixer, and ligand-free UCNPs were precipitated out. The resultant NPs were obtained by centrifugation at $6000 \mathrm{rpm}$ for $20 \mathrm{~min}$, washed once with ethanol and three times with water, and finally redispersed in water. Ligand-free csUCNPs were obtained similar to bare UCNPs, except that OA-csUCNPs in cyclohexane solution were employed.

\section{Preparation of csUCNP/Au nanoassemblies}

For this purpose, $20 \mathrm{mg}$ of bare csUCNPs were dispersed in $4 \mathrm{~mL}$ of $\mathrm{H}_{2} \mathrm{O}$ containing $40 \mathrm{mg}$ of PEI ( $\mathrm{pH} \sim 10$ ), followed by overnight stirring. PEI-modified csUCNPs (abbreviated as PEIcsUCNPs) were obtained via centrifugation at $10000 \mathrm{rpm}$ for $20 \mathrm{~min}$, washed three times with water, and finally redispersed in water and the $\mathrm{pH}$ was adjusted to 5. csUCNP/Au nanoassemblies were prepared by the addition of appropriate freshly prepared $\mathrm{Au}$ NPs to a solution containing PEI-csUCNPs (the content of the csUCNPs was fixed at $0.5 \mathrm{mg} \mathrm{mL}^{-1}$ ) and ultrasonicated for $10 \mathrm{~min}$. Assuming the diameters of the Au NPs and the csUCNPs to be 1.7 and $43 \mathrm{~nm}$, respectively, we can estimate that $\sim 140 \mathrm{Au}$ NPs decorate one csUCNP for $0.10 \mathrm{mM} \mathrm{Au}$ content. The resulting csUCNP/Au nanocomposites were then collected by centrifugation at $9000 \mathrm{rpm}$ for $30 \mathrm{~min}$, washed with water several times, and redispersed in $\mathrm{DMF} / \mathrm{H}_{2} \mathrm{O}(5 / 1$, $\mathrm{v} / \mathrm{v}$ ) solution. This solvent mixture was employed as in pure water the system becomes a bit turbid due to agglomeration processes.

\section{Procedures for cyanide ion sensing}

The sensing of $\mathrm{CN}^{-}$was performed by adding different amounts of $\mathrm{CN}^{-}$solution to the csUCNP/Au nanocomposites in $\mathrm{DMF} / \mathrm{H}_{2} \mathrm{O}(5 / 1, \mathrm{v} / \mathrm{v})$ solution; the concentration of the csUCNPs was kept at $0.5 \mathrm{mg} \mathrm{mL} \mathrm{m}^{-1}$, and the concentration of
$\mathrm{CN}^{-}$ranged from 0 to $280 \mu \mathrm{M}$. Selectivity experiments were performed by the addition of $2 \mathrm{mM}$ of other ions with a similar procedure. The UCL spectra of the resulting samples were recorded under the excitation of a $3 \mathrm{~W} 980 \mathrm{~nm}$ laser.

\section{Results and discussion}

\section{Design principle for cyanide anion sensing}

The design strategy of a csUCNP/Au nanosystem for selective cyanide anion sensing relies on the FRET process, where coreshell-structured upconversion NPs are used as the energy donor and Au NPs are employed as the energy acceptor, as shown in Fig. 1a. Without modification, csUCNPs give rise to green and red luminescence emissions deriving from the ${ }^{2} \mathrm{H}_{11 / 2} \rightarrow{ }^{4} \mathrm{I}_{15 / 2},{ }^{4} \mathrm{~S}_{3 / 2} \rightarrow{ }^{4} \mathrm{I}_{15 / 2}$, and ${ }^{4} \mathrm{~F}_{9 / 2} \rightarrow{ }^{4} \mathrm{I}_{15 / 2}$ transitions of $\mathrm{Er}^{3+}$ under $980 \mathrm{~nm}$ laser excitation. Ultrasmall Au NPs (diameter of $c a .1 .7 \mathrm{~nm}$ ) with a broad absorption in the whole visible region exhibit quenching rather than the plasmonic effect. When the csUCNPs couple with Au NPs, an efficient FRET process occurs, resulting in the weakening of green and red emissions in the UCL intensity. However, the energy transfer from csUCNPs to Au NPs is suppressed in the presence of cyanide ions, owing to the etching property of cyanide ions for $\mathrm{Au}$ NPs. As a consequence, the UCL of csUCNPs is recovered by the consumption of Au NPs upon the addition of cyanide ions (Fig. 1b), and the variation in the UCL intensity of csUCNP/Au nanoassemblies allows the quantitative detection of cyanide ions.

\section{Characterization of csUCNPs/Au nanoassemblies}

Hydrophobic oleate-capped UCNPs were first prepared by employing OA as the ligand via a high-temperature coprecipitation method. ${ }^{45}$ The TEM image shows that the core UCNPs have a uniform hexagonal morphology with an average diameter of about $35 \mathrm{~nm}$ (Fig. 2a), and the average diameter of the hexagonal csUCNPs increases to approximately $43 \mathrm{~nm}$ with uniformity maintained, suggesting the successful growth of the shell on the core NPs (Fig. 2b). Additionally, the XRD pat-
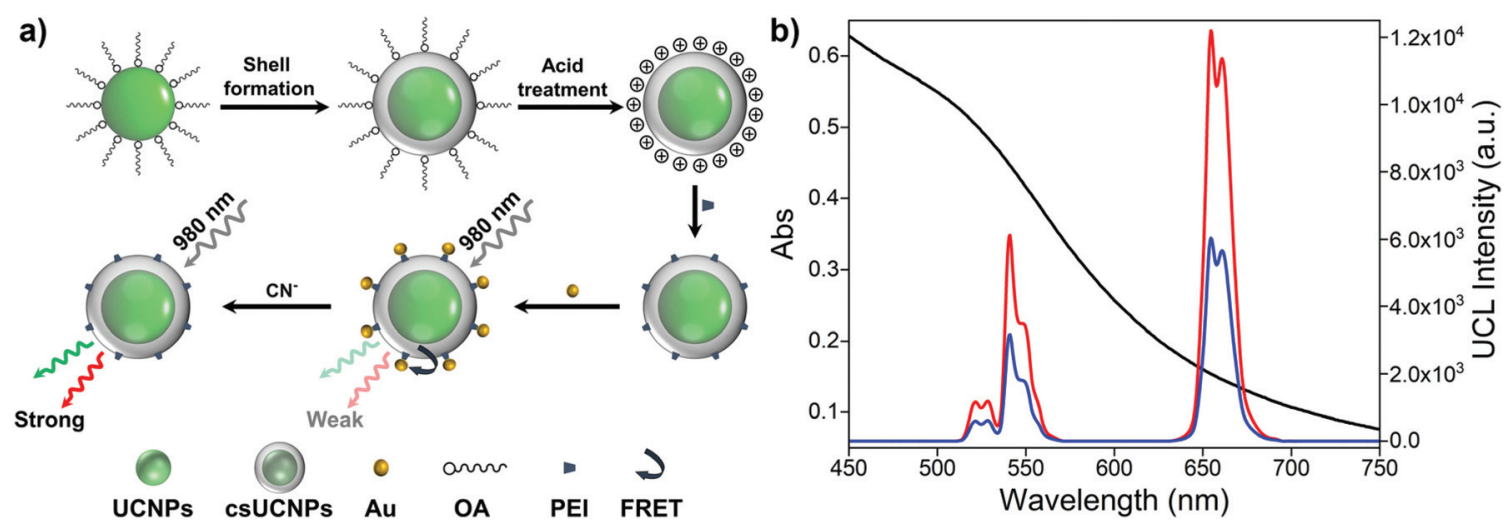

Fig. 1 (a) Schematic illustration of the synthesis of csUCNP/Au nanoassemblies and their response to cyanide ions. (b) UV-vis spectrum of Au NPs (black) and UCL spectra of CsUCNPs/Au in the absence (blue) and presence (red) of cyanide ions. 


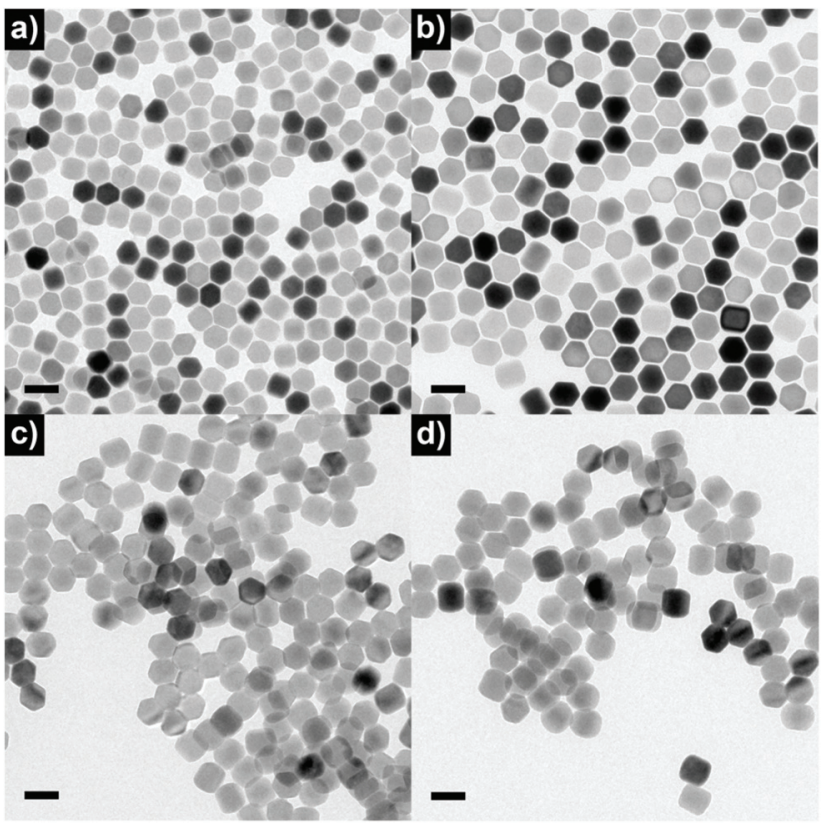

Fig. 2 TEM images of (a) OA-UCNPs, (b) OA-csUCNPs, (c) ligand-free csUCNPs, and (d) PEI-csUCNPs. Scale bars: $50 \mathrm{~nm}$.

terns of the core-only UCNPs and core-shell-structured csUCNPs, which are in excellent agreement with the pure hexagonal phase (JCPDS file number 28-1192), demonstrate high crystallinity with well-defined diffraction peaks (Fig. S1 $\dagger$ ). After the treatment of OA-UCNPs with formic acid, the oleate ligand can be easily removed. ${ }^{47}$ The obtained ligand-free csUCNPs can be well-dispersed in water to form a stable colloidal solution, and the zeta potential was determined to be $36.8 \mathrm{mV}(\mathrm{pH}$ 25.5). Moreover, these well-dispersed bare csUCNPs favor the attachment of PEI on their surface. As revealed by TEM images, the particle size and morphology are retained after ligand removal and further polymer functionalization (Fig. 2c and d).

Additionally, FT-IR measurements were performed to characterize ligand removal and subsequent PEI attachment
(Fig. S2†). Two strong transition bands centered at 2923 and $2854 \mathrm{~cm}^{-1}$ are clearly observed in the OA-csUCNP sample, which can be assigned to the asymmetric and symmetric stretching vibrations of methylene groups in the long alkyl chain, and a weak peak at $3008 \mathrm{~cm}^{-1}$, assigned to the $=\mathrm{C}-\mathrm{H}$ stretching vibration, is shown in the spectrum. Moreover, the two peaks centered at 1561 and $1460 \mathrm{~cm}^{-1}$ can be assigned to the asymmetric and symmetric stretching vibrations of the carboxylate group. These characteristic peaks validate the presence of the oleate ligand on the surface of csUCNPs. However, these characteristic peaks vanish after the formic acid treatment, except for the broad band of solvated water molecules centered at around $3420 \mathrm{~cm}^{-1}$, proving the success in surface ligand removal by acid treatment and confirming the hydrophilic nature of the obtained ligand-free csUCNPs. After polymer functionalization, two bands centered at 2930 and $2854 \mathrm{~cm}^{-1}$ appear, which can be attributed to the asymmetric and symmetric stretching vibrations of the $\mathrm{C}-\mathrm{H}$ bond, respectively. Furthermore, a weak peak centered at $1167 \mathrm{~cm}^{-1}$ attributed to the stretching vibrations of the $\mathrm{C}-\mathrm{N}$ bond and a strong transition band centered at $1545 \mathrm{~cm}^{-1}$ attributed to $\mathrm{N}-\mathrm{H}$ bending vibrations can be observed, thereby revealing the attachment of PEI on the surface of bare csUCNPs.

To obtain csUCNP/Au nanoassemblies, ultrasmall Au NPs with a mean diameter of about $1.7 \mathrm{~nm}$ were prepared (Fig. 3a), and the nanocomposites could then be easily generated by assembling negatively charged $(-2.7 \mathrm{mV}) \mathrm{Au}$ NPs onto the positively charged PEI-csUCNPs $(34.3 \mathrm{mV})$ through electrostatic interactions, as confirmed by TEM (Fig. 3b). As the connection between csUCNP and Au relies on electrostatic interactions, the nonuniform distribution of Au NPs is likely caused by the drying process during the sample preparation for the TEM measurement, as during drying the concentration of the Au NPs increases and therefore their tendency for clustering increases. Compositional analysis of hybrid NPs by EDS shows the presence of $\mathrm{Na}, \mathrm{F}, \mathrm{Y}, \mathrm{Yb}$, and Au elements (Fig. 3c), further evidencing the presence of Au NPs. The hydrodynamic radius of $0.5 \mathrm{mg} \mathrm{mL}^{-1}$ csUCNP/Au (0.1 mM Au NPs) nanocomposites remained stable at $\sim 135 \mathrm{~nm}$ for a period of at least
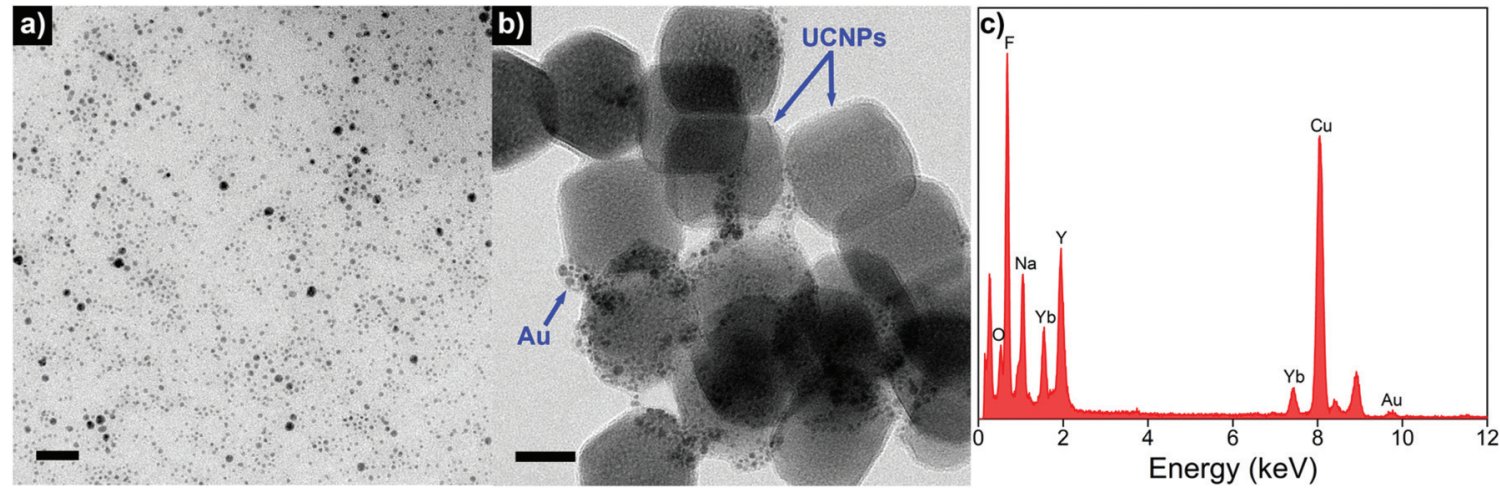

Fig. 3 TEM images of (a) Au NPs and (b) csUCNP/Au nanoassemblies. Scale bars: $20 \mathrm{~nm}$. (c) EDS spectrum of csUCNP/Au nanoassemblies. 

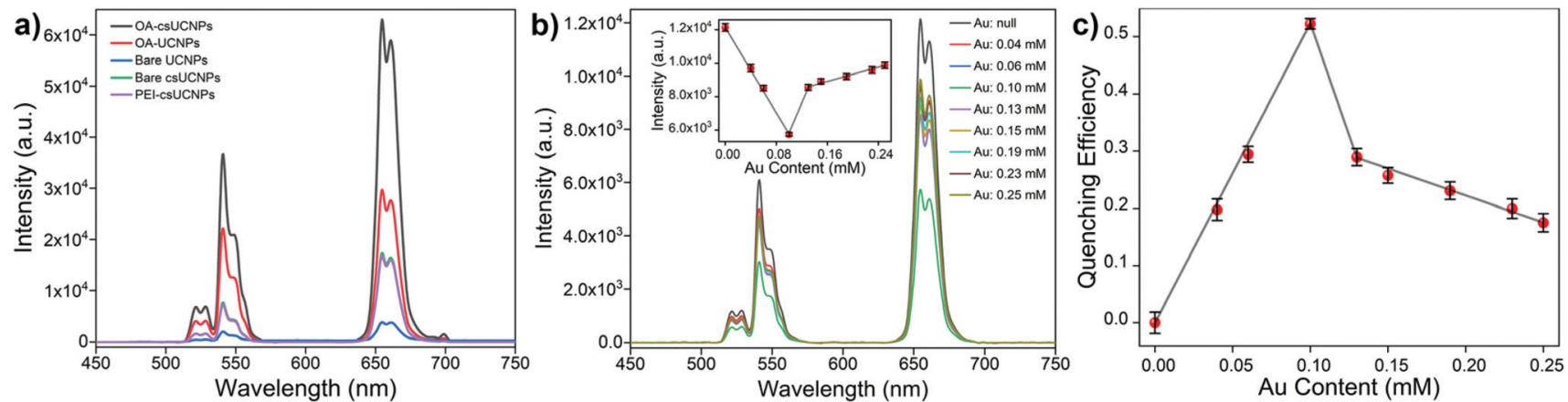

Fig. 4 (a) UCL spectra of OA-UCNPs and OA-csUCNPs dispersed in cyclohexane, bare UCNPs, bare csUCNPs, and PEI-csUCNPs dispersed in water with a concentration of $1 \mathrm{mg} \mathrm{mL}^{-1}$ under $3 \mathrm{~W} 980 \mathrm{~nm}$ excitation. (b) UCL spectra of $0.5 \mathrm{mg} \mathrm{mL}^{-1} \mathrm{csUCNPs}$ upon the addition of different contents of Au NPs. Inset: Variations of UCL intensity at $655 \mathrm{~nm}$ upon the addition of Au NPs. (c) Relationship between the fluorescence quenching efficiency of csUCNP/Au nanoassemblies in the $600-700 \mathrm{~nm}$ range and the Au content. The black line serves as a guide to the eye. Error bars represent the standard deviations of three independent measurements.

14 days (Fig. S3†), exhibiting excellent stability of the nanocomposites dissolved in the mixed solution.

Next, the optical properties of the csUCNP and csUCNP/Au nanocomposites are investigated. The UCL emission spectra of hydrophobic OA- and ligand-free upconversion NPs under $980 \mathrm{~nm}$ laser excitation are shown in Fig. 4a, presenting a relatively low UCL intensity of the OA-UCNPs due to the energy loss by large surface defects. ${ }^{48}$ Here a remarkable loss of green and red emissions in UCL intensity is seen in aqueous solution after the transition of OA-UCNPs to ligand-free ones via ligand exfoliation by formic acid, attenuated by water molecules. ${ }^{49,50}$ In order to enhance the UCL intensity of upconversion NPs, especially hydrophilic ones, core-shellstructured OA-csUCNPs were prepared via the same synthetic procedure by coating a similar material on core OA-UCNPs, ${ }^{46}$ and bare csUCNPs were obtained via the same vortexing method. The UCL intensity of OA-csUCNPs increases by a factor of two compared with the core-only OA-UCNPs in cyclohexane, whereas the UCL intensity of bare csUCNPs is about 4.5 times larger than that of core-only ligand-free UCNPs in aqueous solution, owing to passivated surface defects by the shell layer. Meanwhile, nearly identical UCL emissions of PEIand bare csUCNPs are observed.

\section{Fluorescence quenching ability of Au NPs to csUCNPs}

The optical properties of Au NPs were assessed by UV-vis absorption spectroscopy. Au NPs show a broad absorption band in the visible region. The broad spectral absorbance of $\mathrm{Au}$ NPs overlaps well with the UCL of csUCNPs in the green and red regions, as shown in Fig. 1b, enabling efficient FRET between csUCNPs and Au NPs. Furthermore, the FRET process is verified by the phenomenon of UCL reduction in csUCNPs/ Au upon $980 \mathrm{~nm}$ laser activation.

To optimize the nanosystem, different contents of Au NPS were used to modify PEI-csUCNPs. The performance of different amounts of Au NPs on PEI-UCNPs is evaluated from UCL spectra, which are shown in Fig. 4b. The UCL intensity experiences a significant decrease at the beginning and reaches a minimum when a $0.1 \mathrm{mM}$ Au NP solution is added. However, at higher Au content this trend suddenly stops, and an increase in UCL intensity occurs when the content of Au NPs goes beyond $0.1 \mathrm{mM}$. Subsequently, a gradual upturn in UCL intensity occurs upon further increasing the Au NP content. In addition, the energy transfer efficiency between
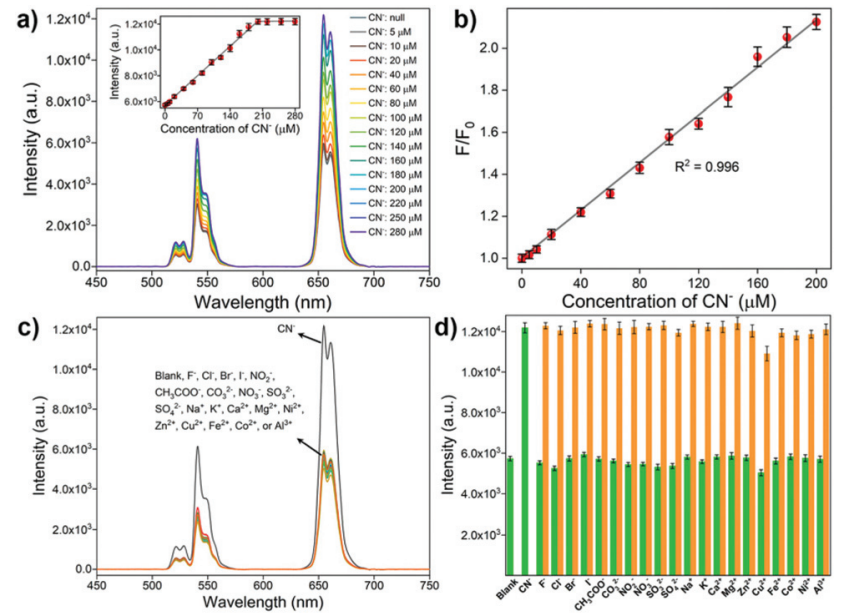

Fig. 5 (a) UCL spectra of $0.5 \mathrm{mg} \mathrm{mL}^{-1}$ csUCNPs/Au (0.1 mM Au NPs) upon the addition of different concentrations of cyanide ions. Inset: $\mathrm{UCL}$ intensity at $655 \mathrm{~nm}$ as a function of the cyanide ion concentration. (b) Linear relationship between the UCL enhancement factor $\left(F / F_{0}\right)$ at $655 \mathrm{~nm}$ and the concentration of cyanide ions, where $F_{0}$ and $F$ are the luminescence intensities of nanoprobes at $655 \mathrm{~nm}$ in the absence and presence of cyanide ions, respectively. (c) $U C L$ spectra of $0.5 \mathrm{mg} \mathrm{mL}^{-1}$ csUCNP/Au nanocomposites in the presence of various anions. (d) Changes in UCL intensity of $0.5 \mathrm{mg} \mathrm{mL}^{-1}$ csUCNP/Au at $655 \mathrm{~nm}$ upon the addition of $200 \mu \mathrm{M} \mathrm{CN}^{-}$and $2 \mathrm{mM}$ other ions. Green bars represent the UCL changes upon the addition of various ions, and the orange bars represent the subsequent addition of $200 \mu \mathrm{M} \mathrm{CN}^{-}$to the above solution. Error bars represent the standard deviations of three independent measurements. 
csUCNPs and Au NPs (i.e., quenching efficiency) is calculated from the UCL emission intensities in the red region (600-700 nm) using the following equation:

$$
\eta=1-\mathrm{rUCL} / \mathrm{rUCL}_{0}
$$

where $\eta$ denotes the quenching efficiency in the red region, and rUCL and $\mathrm{rUCL}_{0}$ are the integrated red emission intensities of csUCNPs/Au and csUCNPs, respectively, in a 600-700 $\mathrm{nm}$ range.

As shown in Fig. 4c, the UCL quenching efficiency in a wavelength range of 600-700 $\mathrm{nm}$ experiences a substantial increase for a low loading amount of Au NPs, and the greatest quenching efficiency of about $52 \%$ is reached upon the addition of $0.1 \mathrm{mM} \mathrm{Au}$ NPs. For higher $\mathrm{Au}$ content, the quenching efficiency decreases with the increasing content of $\mathrm{Au}$ NPs. This abnormal phenomenon for the high loading amount of Au NPs may be attributed to the partial aggregation of Au NPs on csUCNPs, leading to the plasmonic enhancement effect.

In order to verify the aforementioned conjecture, TEM and UV-vis absorption measurements of csUCNP/Au nanocomposites were performed. Obviously, TEM images show that no apparent aggregation of Au NPs takes place for low amounts of added $\mathrm{Au}$ NPs (Fig. S4a-c $\dagger$ ). In contrast, small agglomerates appear when $0.13 \mathrm{mM}$ Au NPs are added, and the agglomerates become larger with the increasing loading amount of Au NPs (Fig. S4d-h†). Moreover, no obvious absorption peak is observed when the added amount of Au NPs is less than or equal to $0.1 \mathrm{mM}$. However, an absorption peak at about $535 \mathrm{~nm}$ appears when the additive amount of Au NPs is greater than $0.1 \mathrm{mM}$, and the absorption intensity at $535 \mathrm{~nm}$ increases with the growing addition of Au NPs to PEI-csUCNP solutions, indicating the increasing amount of Au aggregates (Fig. S4i†). As a consequence, the competition of the Au-aggregation-induced plasmonic enhancement effect ${ }^{51}$ and single ultrasmall $\mathrm{Au}$ NP-induced quenching effect results in the uptrend of the UCL intensity in csUCNP/Au nanoassemblies with a high content of Au NPs.

\section{Sensitive and selective detection of cyanide ions}

The sensing ability of csUCNP/Au nanoassemblies is based on the recovery of UCL emissions via the consumption of Au NPS by the addition of cyanide ions, attributed to the cyanide- mediated oxidation reaction of $\mathrm{Au}$ into $\left[\mathrm{Au}(\mathrm{CN})_{2}\right]^{-}$, as described by the following equation:

$$
4 \mathrm{Au}+8 \mathrm{CN}^{-}+\mathrm{O}_{2}+2 \mathrm{H}_{2} \mathrm{O} \rightarrow 4\left[\mathrm{Au}(\mathrm{CN})_{2}\right]^{-}+4 \mathrm{OH}^{-}
$$

The csUCNP/Au nanoassemblies formed by the addition of $0.1 \mathrm{mM}$ Au NPs are applied for the sensing of cyanide ions due to their sufficient fluorescence quenching efficiency. As discussed above, the consumption of Au NPs occurs upon the addition of cyanide ions, resulting in the suppression of the FRET process and corresponding to a gradual increase of UCL intensity in all emissions, as shown in Fig. 5a. No further variation of UCL intensity is observed after the addition of 2 equiv. of cyanide ions, as expected from the reaction equation. The variations of the fluorescence enhancement factor at $655 \mathrm{~nm}$ ( $F / F_{0}$, where $F_{0}$ and $F$ refer to the luminescence intensities of nanoprobes at $655 \mathrm{~nm}$ in the absence and presence of cyanide ions, respectively) versus the addition of cyanide ions are shown in Fig. 5b. The calibration curve exhibits a linear relationship between $F / F_{0}$ and the concentration of cyanide ions in a range from 0 to $200 \mu \mathrm{M}$, and the linear correlation coefficient of the calibration curve is 0.996 . The limit of detection (LOD) for cyanide is calculated to be $1.53 \mu \mathrm{M}$ according to the $3 \sigma$ rule. Compared to the developed methods for cyanide ion sensing (Table 1), the sensitivity of the UCNP/Au nanocomposites is well competitive.

To further assess the selectivity of the nanosystem for cyanide ions, a series of control experiments was performed to investigate the response of csUCNPs/Au toward other potentially interfering ions. As shown in Fig. 5c, only the addition of cyanide ions leads to a noticeable variation in UCL, but no obvious UCL changes are observed upon the addition of other ions, such as $\mathrm{F}^{-}, \mathrm{Cl}^{-}, \mathrm{Br}^{-}, \mathrm{I}^{-}, \mathrm{CH}_{3} \mathrm{COO}^{-}, \mathrm{CO}_{3}{ }^{2-}, \mathrm{NO}_{2}{ }^{-}, \mathrm{NO}_{3}{ }^{-}$, $\mathrm{SO}_{3}{ }^{2-}, \mathrm{SO}_{4}{ }^{2-}, \mathrm{Na}^{+}, \mathrm{K}^{+}, \mathrm{Ca}^{2+}, \mathrm{Mg}^{2+}, \mathrm{Zn}^{2+}, \mathrm{Cu}^{2+}, \mathrm{Fe}^{2+}, \mathrm{Ni}^{2+}, \mathrm{Co}_{2}{ }^{+}$, and $\mathrm{Al}^{3+}$. In addition, competition experiments were carried out by adding cyanide anions to solutions of csUCNPs/Au in the presence of other ions. Significant enhancements of the UCL intensity are observed upon the addition of cyanide ions, as shown in Fig. 5d. Therefore, it can be stated that csUCNP/ $\mathrm{Au}$ nanoassemblies can act as highly sensitive and selective fluorescence nanoprobes for cyanide ion sensing.

Table 1 Comparison of various methods for cyanide ion sensing

\begin{tabular}{llll}
\hline Materials & Methods & Linear range & LOD \\
\hline Gold nanocluster & Fluorometry & $0-10 \mu \mathrm{M}$ & $0.2 \mu \mathrm{M}$ \\
Iridium-complex-modified UCNPs & Fluorometry & $0-1.8 \mathrm{mM}$ & $62.6 \mu \mathrm{M}$ \\
Phenothiazine-cyanine-modified UCNPs & Colorimetry & $0-50 \mu \mathrm{M}$ & 42 \\
& Fluorometry & $0-300 \mathrm{uM}$ & $9.85 \mu \mathrm{M}$ \\
Bicinchoninic acid-Cu(II) complex & Colorimetry & $0-50 \mathrm{mM}$ & $0.84 \mu \mathrm{M}$ \\
Co(II)Pc modified carbon paste electrode & Voltammetry & $24-270 \mu \mathrm{M}$ & $4.25 \mu \mathrm{M}$ \\
Chitosan-gold nanoparticle & Colorimetry & $0-38 \mu \mathrm{M}$ & $3.6 \mu \mathrm{M}$ \\
Hexaazatriphenylene & Electrochemical assay & $0-200 \mu \mathrm{M}$ & $2.31 \mu \mathrm{M}$ \\
csUCNP/Au nanocomposites & Fluorometry & $0.87 \mu \mathrm{M}$ & 53 \\
& & $0-200 \mu \mathrm{M}$ & 54 \\
\end{tabular}




\section{Conclusions}

In summary, we have developed a facile approach to prepare csUCNP/Au nanoassemblies for cyanide ion sensing based on the FRET process, where csUCNPs act as the energy donor and $\mathrm{Au}$ NPs act as the energy acceptor. For this purpose, the optimum Au NP loading content was determined with the aim of achieving the highest fluorescence quenching efficiency. Au NPs are exclusively etched by cyanide ions but are inert to other ions, and the cyanide-induced Au consumption favors the inhibition of the FRET process, leading to the recovery of the UCL emissions. Competition experiments show that the response of csUCNP/Au nanoassemblies toward cyanide ions remains undisturbed by the presence of other interfering ions. This means that the method presented here allows for the selective sensing of cyanide ions in the presence of other ions with high sensitivity $(1.53 \mu \mathrm{M})$. Such a cyanide-mediated FRET process may be extended to the future design of other upconversion-based analysts.

\section{Author contributions}

C. Sun and M. Gradzielski conceived the concepts. C. Sun conducted the experiments and analysed the data. M. Gradzielski supervised the entire process, and both authors discussed the results and jointly wrote the manuscript.

\section{Conflicts of interest}

The authors declare no competing financial interest.

\section{Acknowledgements}

C. Sun acknowledges the financial support from the China Scholarship Council (CSC, No. 201404910463) and TU Berlin. The authors are grateful to Ina Speckmann and Jan Ron Justin Simke for the XRD and TEM measurements, respectively.

\section{Notes and references}

1 Z.-Z. Dong, C. Yang, K. Vellaisamy, G. Li, C.-H. Leung and D.-L. Ma, ACS Sens., 2017, 2, 1517-1522.

2 K. Deng, L. Wang, Q. Xia, R. Liu and J. Qu, Sens. Actuators, $B, 2019,296,126645$.

3 J. Ma and P. K. Dasgupta, Anal. Chim. Acta, 2010, 673, 117125.

4 R. R. Dash, A. Gaur and C. Balomajumder, J. Hazard. Mater., 2009, 163, 1-11.

5 Y. G. Timofeyenko, J. J. Rosentreter and S. Mayo, Anal. Chem., 2007, 79, 251-255.

6 G. Ding, H. Zhou, J. Xu and X. Lu, Chem. Commun., 2014, 50, 655-657.
7 A. Safavi, N. Maleki and H. R. Shahbaazi, Anal. Chim. Acta, 2004, 503, 213-221.

8 M. Tomasulo, S. Sortino, A. J. P. White and F. M. Raymo, J. Org. Chem., 2006, 71, 744-753.

9 D. Senapati, S. S. R. Dasary, A. K. Singh, T. Senapati, H. Yu and P. C. Ray, Chem. - Eur. J., 2011, 17, 8445-8451.

10 G. Liu, W. Cai, L. Kong, G. Duan, Y. Li, J. Wang and Z. Cheng, J. Hazard. Mater., 2013, 248-249, 435-441.

11 R. Badugu, J. R. Lakowicz and C. D. Geddes, J. Am. Chem. Soc., 2005, 127, 3635-3641.

12 Y. Liu, K. Ai, X. Cheng, L. Huo and L. Lu, Adv. Funct. Mater., 2010, 20, 951-956.

13 M. Haase and H. Schäfer, Angew. Chem., Int. Ed., 2011, 50, 5808-5829.

14 S. Gai, C. Li, P. Yang and J. Lin, Chem. Rev., 2014, 114, 2343-2389.

15 G.-R. Tan, M. Wang, C.-Y. Hsu, N. Chen and Y. Zhang, Adv. Opt. Mater., 2016, 4, 984-997.

16 N. Wang, X. Yu, K. Zhang, C. A. Mirkin and J. Li, J. Am. Chem. Soc., 2017, 139, 12354-12357.

17 R. Wei, Z. Wei, L. Sun, J. Z. Zhang, J. Liu, X. Ge and L. Shi, ACS Appl. Mater. Interfaces, 2016, 8, 400-410.

18 J. Peng, W. Xu, C. L. Teoh, S. Han, B. Kim, A. Samanta, J. C. Er, L. Wang, L. Yuan, X. Liu and Y.-T. Chang, J. Am. Chem. Soc., 2015, 137, 2336-2342.

19 Y. Zhou, W. Pei, C. Wang, J. Zhu, J. Wu, Q. Yan, L. Huang, W. Huang, C. Yao, J. S. C. Loo and Q. Zhang, Small, 2014, 10, 3560-3567.

20 C. Li, J. Liu, S. Alonso, F. Li and Y. Zhang, Nanoscale, 2012, 4, 6065-6071.

21 Y. Zhou, W. Pei, X. Zhang, W. Chen, J. Wu, C. Yao, L. Huang, H. Zhang, W. Huang, S. C. J. Loo and Q. Zhang, Biomaterials, 2015, 54, 34-43.

22 J. Peng, C. L. Teoh, X. Zeng, A. Samanta, L. Wang, W. Xu, D. Su, L. Yuan, X. Liu and Y.-T. Chang, Adv. Funct. Mater., 2016, 26, 191-199.

23 M. K. Mahata and K. T. Lee, Nanoscale Adv., 2019, 1, 23722381.

24 Q. Liu, J. Peng, L. Sun and F. Li, ACS Nano, 2011, 5, 80408048.

25 X. Li, Y. Wu, Y. Liu, X. Zou, L. Yao, F. Li and W. Feng, Nanoscale, 2014, 6, 1020-1028.

26 Y. Liu, A. Jiang, Q. Jia, X. Zhai, L. Liu, L. Ma and J. Zhou, Chem. Sci., 2018, 9, 5242-5251.

27 L. Wu, J. Wang, M. Yin, J. Ren, D. Miyoshi, N. Sugimoto and X. Qu, Small, 2014, 10, 330-336.

28 C. Zhang, Y. Yuan, S. Zhang, Y. Wang and Z. Liu, Angew. Chem., Int. Ed., 2011, 50, 6851-6854.

29 C. Liu, Z. Wang, H. Jia and Z. Li, Chem. Commun., 2011, 47, 4661-4663.

30 P. Vilela, A. El-Sagheer, T. M. Millar, T. Brown, O. L. Muskens and A. G. Kanaras, ACS Sens., 2016, 2, 52-56.

31 Y. Xiao, L. Zeng, T. Xia, Z. Wu and Z. Liu, Angew. Chem., Int. Ed., 2015, 54, 5323-5327.

32 X. Chen, J. Wang, C. Yang, Z. Ge and H. Yang, Sens. Actuators, B, 2017, 255, 1316-1324. 
33 M. Wang, W. Hou, C.-C. Mi, W.-X. Wang, Z.-R. Xu, H.-H. Teng, C.-B. Mao and S.-K. Xu, Anal. Chem., 2009, 81, 8783-8789.

34 L. Liu, R. Li, G. Wang, Z. Gu and Z. Li, Sens. Actuators, B, 2019, 285, 453-461.

35 H. Kurt, M. Yüce, B. Hussain and H. Budak, Biosens. Bioelectron., 2016, 81, 280-286.

36 L. Xu, J. Liu, L. Pei, Y. Xu and Z. Xia, J. Mater. Chem. C, 2019, 7, 6112-6119.

37 Y. Cen, J. Tang, X.-J. Kong, S. Wu, J. Yuan, R. Yu and X. Chu, Nanoscale, 2015, 7, 13951-13957.

38 R. Deng, X. Xie, M. Vendrell, Y.-T. Chang and X. Liu, J. Am. Chem. Soc., 2011, 133, 20168-20171.

39 J. Yuan, Y. Cen, X.-J. Kong, S. Wu, C.-L. Liu, R.-Q. Yu and X. Chu, ACS Appl. Mater. Interfaces, 2015, 7, 10548-10555.

40 Y. Yuan, S. Wu, F. Shu and Z. Liu, Chem. Commun., 2014, 50, 1095-1097.

41 J. Liu, Y. Liu, Q. Liu, C. Li, L. Sun and F. Li, J. Am. Chem. Soc., 2011, 133, 15276-15279.

42 L. Yao, J. Zhou, J. Liu, W. Feng and F. Li, Adv. Funct. Mater., 2012, 22, 2667-2672.

43 S. Zhao, F. Wu, Y. Zhao, Y. Liu and L. Zhu, J. Photochem. Photobiol., A, 2016, 319-320, 53-61.

44 D. G. Duff, A. Baiker and P. P. Edwards, Langmuir, 1993, 9, 2301-2309.
45 Z. Li and Y. Zhang, Nanotechnology, 2008, 19, 345606.

46 F. Wang, R. Deng, J. Wang, Q. Wang, Y. Han, H. Zhu, X. Chen and X. Liu, Nat. Mater., 2011, 10, 968973.

47 C. Sun, J. R. J. Simke and M. Gradzielski, Mater. Adv., 2020, 1, 1602-1607.

48 F. Wang, J. Wang and X. Liu, Angew. Chem., Int. Ed., 2010, 49, 7456-7460.

49 R. Arppe, I. Hyppanen, N. Perala, R. Peltomaa, M. Kaiser, C. Wurth, S. Christ, U. Resch-Genger, M. Schaferling and T. Soukka, Nanoscale, 2015, 7, 11746-11757.

50 F. T. Rabouw, P. T. Prins, P. Villanueva-Delgado, M. Castelijns, R. G. Geitenbeek and A. Meijerink, ACS Nano, 2018, 12, 4812-4823.

51 L. Polavarapu, J. Pérez-Juste, Q.-H. Xu and L. M. LizMarzán, J. Mater. Chem. C, 2014, 2, 74607476.

52 A. A. Biradar, A. V. Biradara, T. Sun, Y. Chan, X. Huang and T. Asefa, Sens. Actuators, B, 2016, 222, 112-119.

53 E. Figueira, L. Neres, M. Ruy, G. Troiano and M. D. Sotomayor, Anal. Methods, 2016, 8, 6353-6360.

54 C. Radhakumary and K. Sreenivasan, Analyst, 2012, 137, 5387-5391.

55 G. Men, W. Han, C. Chen, C. Liang and S. Jiang, Analyst, 2019, 144, 2226-2230. 